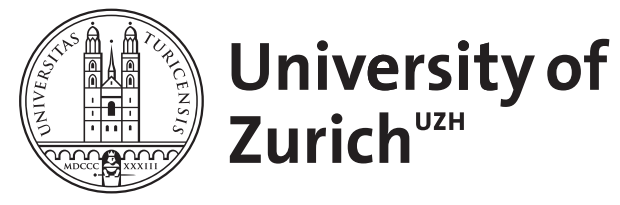

What's in a name? Atopic dermatitis or atopic eczema, but not eczema alone

Silverberg, J I ; Thyssen, J P ; Paller, A S ; Drucker, A M ; Wollenberg, A ; Lee, K H ; Kabashima, K ; Todd, G ; Schmid-Grendelmeier, P ; Bieber, T

DOI: https://doi.org/10.1111/all.13225

Posted at the Zurich Open Repository and Archive, University of Zurich ZORA URL: https://doi.org/10.5167/uzh-140844

Journal Article

Accepted Version

Originally published at:

Silverberg, J I; Thyssen, J P; Paller, A S; Drucker, A M; Wollenberg, A; Lee, K H; Kabashima, K; Todd, G; Schmid-Grendelmeier, P; Bieber, T (2017). What's in a name? Atopic dermatitis or atopic eczema, but not eczema alone. Allergy, 72(12):2026-2030.

DOI: https://doi.org/10.1111/all.13225 
DOI: 10.1111 /all.13225

\title{
What's in a name? Atopic dermatitis or atopic eczema, but not eczema alone
}

\author{
J. I. (D) | J. P. Thyssen ${ }^{2}$ | A. S. Paller ${ }^{3}$ A. M. Drucker ${ }^{4}$ | A. Wollenberg ${ }^{5}$ | \\ K. H. Lee ${ }^{6} \mid$ K. Kabashima ${ }^{7}$ G. Todd ${ }^{8} \mid$ P. Schmid-Grendelmeier ${ }^{9,10} \mid$ T. Bieber ${ }^{11}$
}

\author{
'Departments of Dermatology, Preventive Medicine and Medical Social Sciences, Northwestern University Feinberg School of Medicine, Chicago, IL, USA \\ ${ }^{2}$ Department of Dermatology and Allergy, Herlev-Gentofte Hospital, University of Copenhagen, Hellerup, Denmark \\ ${ }^{3}$ Departments of Dermatology and Pediatrics, Northwestern University Feinberg School of Medicine and the Ann and Robert H. Lurie Children's Hospital \\ of Chicago, Chicago, IL, USA \\ ${ }^{4}$ Department of Dermatology, Alpert Medical School of Brown University, Providence, RI, USA \\ ${ }^{5}$ Department of Dermatology and Allergy, Ludwig-Maximilian-University Munich, Munich, Germany \\ ${ }^{6}$ Department of Dermatology and Cutaneous Biology Research Institute, Yonsei University College of Medicine, Seoul, South Korea \\ ${ }^{7}$ Department of Dermatology, Kyoto University Graduate School of Medicine, Kyoto, Japan \\ ${ }^{8}$ Department of Medicine, Faculty of Health Sciences, University of Cape Town, Cape Town, South Africa \\ ${ }^{9}$ Christine KGhne-Center for Allergy Research and Education (CK-CARE) Davos-Augsburg-Bonn-St Gallen-Z目ich, St Gallen, Switzerland \\ ${ }^{10}$ Allergy Unit, Department of Dermatology, University Hospital, Zurich, Switzerland \\ ${ }^{11}$ Department of Dermatology and Allergy, University of Bonn, Germany, and Christine K氏hne-Center for Allergy Research and Education, Davos, Switzerland
}

\section{Correspondence}

Jonathan I. Silverberg, Departments of Dermatology, Preventive Medicine and Medical Social Sciences, Northwestern University Feinberg School of Medicine, Chicago, IL, USA.

Email: JonathanISilverberg@Gmail.com

Edited by: Thomas Bieber

\section{Abstract}

Background: The ideal nomenclature of atopic dermatitis (AD) / atopic eczema (AE) has long been contested. However, it is becoming increasingly clear that the disparate nomenclature of this disease may have important deleterious ramifications for clinical care and research.

Materials and Methods: An electronic questionnaire regarding the preferred nomenclature for $A D$ was sent to councilors of the International Eczema Council (IEC) $(n=77)$, an international group of clinicians and researchers with expertise in $A D / A E$. The survey consisted of 2 questions for consensus regarding the preference for an atopic prefix, and preference for the term $A D$ or $A E$, and an exploratory question about the acceptability of the terms $A D, A E$ or eczema. Consensus was defined a priori as at least $90 \%$ agreement for each question with a response rate of at least $90 \%$.

Results: Seventy-one of 77 (92.2\%) IEC councilors and associates responded to the survey, with all respondents completing the entire survey. Consensus was reached for question 1, with 69 of 71 respondents (97.2\%) preferring the atopic prefix. However, consensus was not reached for question 2, with 40 respondents (58.0\%) preferring the term $A D$ and $30(43,5 \%)$ preferring $A E$. Sixty-three respondents (88.7\%) and 55 (77.5\%) felt that the terms AD and AE were acceptable, whereas only 11 (15.5\%) felt that eczema was acceptable. 
Conclusions: The IEC noted that the term eczema is imprecise, and its use is confusing. The consensus of the IEC was to recommend use of the prefix "atopic" (i.e., $\mathrm{AD}$ or $\mathrm{AE}$ ) in all publications, presentations and discussions about the disorder.

KEYW ORDS

atopic dermatitis, atopic eczema, nomenclature, terminology, consensus

\section{1 | INTRODUCTION}

Atopic dermatitis and atopic eczema ( $A D$ and $A E$ ) are terms that describe a common, clinically defined, pruritic, inflammatory skin condition, characterized by a chronic and relapsing dermatitis in typical anatomical sites. The ideal nomenclature of $A D / A E$ has long been contested. However, it is becoming increasingly clear that the disparate nomenclature of this disease may have important deleterious ramifications for clinical care and research.

\section{1 | Historical aspects of AD/AE nomenclature}

The criteria and terms used to denote AD/AE were recently reviewed. ${ }^{1}$ The term "eczema" (Greek for boiling) was introduced to the medical literature by RobertWilan(1757-1812) as a subgroup of the vesicular (or bullous) disorders and represents a description of primary lesions instead of a disease or etiology. Recurrent and often passionate discussions about the exact definition of eczema have occurred for centuries. $^{2}$

The "Oxford History of English" postulates that the term "dermatitis" was first included in the Oxford English Dictionary only in $1876 .{ }^{3}$ Other names used for AD at that time included "porrigo larvalis," "lichen agrius," and "prurigo Besnier". Atopic eczema (AE) was first used by Abraham Walzer (1883-1965) in 1928. In 1933, Sulzberger and Wise first coined "atopic dermatitis" as a footnote in the "1 933 Year Book of Dermatology and Syphilology".

The term "atopic" was included in the terminology as it emerged that some individuals with $\mathrm{AD} / \mathrm{AE}$ reacted on the skin to allergens. Further, the term "atopy" (Greek for "out of place") was introduced byCoca and Cooke in 1923 as part of a new definition on hypersensitivities, categorized with abnormal immunological patterns. However, the definition of the term atopy is controversial. Some decades ago, atopy was defined by the associated diseases, as an "inheritable tendency to develop the atopic diseases, namely hay fever, bronchial asthma, and atopic dermatitis." Presently, the World Allergy Organization defines atopy as "a personal and/or familial tendency, usually in childhood or adolescence, to become sensitized and produce Immunoglobulin E antibodies in response to ordinary exposure to allergens, usually proteins." Given the lack of a clear definition, some prefer to exclude the term "atopy" and use "eczema" as the standalone disease nomenclature.

A major question has been whether dermatitis, eczema, and other terminology represent the same entity. A pioneering description of AD/AE or "constitutional prurigo" was provided by Hebra as a chronic, recurrent skin disorder, characterized by intensely pruritic papules and nodules on the trunk and limbs. ${ }^{4}$ Later, it was accepted that infantile eczema and neurodermatitis/diathetic prurigo in children and adults represented the same disease. During the second half of the twentieth century, the term "constitutional eczema" was preferred by French dermatologists, "neurodermatitis" by Germanspeaking dermatologists, and "atopic eczema" by British dermatologists. ${ }^{4}$ Presently, many use the terms dermatitis, $A D$, and eczema synonymously, whereas others use the term eczema as a clinical description of the morphology of AD/AElesions, which encompasses the acute, subacute, and chronic ill-defined lesions present in $\mathrm{AD} / \mathrm{AE}$.

A recent consensus statement by the International League of Dermatological Societies, however, did not recognize eczema as an official morphological term. ${ }^{5}$ The disparate nomenclature used for $\mathrm{AD} / \mathrm{AE}$ might introduce confusion and bias in reporting, as reviewed below. Hence, there is a need for a common nomenclature when discussing disease entities such as $\mathrm{AD} / \mathrm{AE}$.

\subsection{Recent trends in the use of terminology}

Some interesting trends of terminology have emerged for AD over the past few decades as shown in a recent review article. ${ }^{6}$ Publications using any of the different terms for $A D / A E$ increased over the past 60 years, ${ }^{6}$ reflecting a combination of the increasing AD/ $A E$ prevalence worldwide, ${ }^{7-9}$ the growing interest in the clinical features and scientific basis of $A D / A E$, and increases in resources, technologies, and the ability for scientific communication internationally. The use of the terms "atopic dermatitis" and "atopic eczema" particularly increased in the late 1970s, coinciding with discussion of the seminal classification for AD/AE at the International Symposium of Atopic Dermatitis in Oslo in 1979, followed by the publication of the Hanifin and Rajka criteria for AD/AE. ${ }^{10}$ Publications using the term AD continue to increase annually, while publications using $A E$ have slightly decreased annually between 2008 and 2015

\section{3 | Current use of terminology}

\subsection{1 | Scientificliterature}

Of the many terms used for $A D / A E$ in the scientific literature (Table 1), a recent systematic review and meta-analysis found that 
TABLE 1 Different terms used for AD in the scientific literature Atopic dermatitis

Atopic eczema

Atopic neurodermatitis

Atopiform dermatitis

Besnier's prurigo

Childhood eczema

Dermatitis

Eczema

Flexural eczema

Infantile eczema

Lichen agrius

Neurodermatitis

Porrigo larvalis

"atopic dermatitis" and "eczema" were the most common. ${ }^{6}$ In MEDLINE (PubMed), $64.4 \%$ of publications addressing AD/AE used the term "atopic dermatitis," $46.9 \%$ "eczema" and only $7.5 \%$ "atopic eczema." Few publications used the terminology "childhood eczema," "flexural eczema," "infantile eczema," "atopic neurodermatitis," or "Besnier's prurigo." AD was also the most frequently used term in studies of adults and children, humans and animals, males and females, followed by "eczema" alone. AD was the most commonly used term in the highest impact-factor dermatology and allergy/immunology journals, whereas "eczema" was the most commonly used term in the pediatric and internal medicine literature. "Atopic eczema" was the least commonly used of the three terms in all of these subgroups. It is imperative that harmonization of the nomenclature for $A D$ includes the many different specialties that manage $\mathrm{AD}$ and the broader healthcare provider community.

There appear to be regional differences with respect to the preferred terminology. AD was the most commonly used term in publications written in English, Japanese, Spanish, ${ }^{6}$ and Korean (unpublished data), whereas "eczema" was most often used in publications written in French, German, Italian, and Russian. "Atopic eczema" was the least commonly used of the three terms in all of these languages. These regional differences underscore the imperative for international consensus on $A D$ nomenclature.

\subsection{2 | Laypopulation}

"Eczema" is the dominant terminology used by the lay population. ${ }^{11}$ A recent study found that "eczema" and its common misspellings accounted for $84 \%$ of combined monthly searches on the Google, Bing, Yahoo, and Baidu Internet search engines. ${ }^{11} \mathrm{AD}$ and $\mathrm{AE}$ accounted for only $14 \%$ and $2 \%$ of searches, respectively. Again, there appeared to be considerable regional differences in the popularity of terminology. "Eczema" was the most popular terminology searched for in English, Russian, Turkish, and Japanese, and "atopic eczema" was the least popular of the three terms by far. Consistent with this trend, the patient organizations for AD/AE in the USA and
UK are titled the National Eczema Association and National Eczema Society, respectively, and even the International Eczema Council was so named to be understood by the lay public.

\subsection{3 | Clinical terminology}

Disparate terms are also used for AD/AE in the clinical setting, which has a number of important and potentially problematic ramifications. Many clinicians treat the term "eczema" as a morphological descriptor for characteristic cutaneous lesions and histologic alterations in biopsy sections; this is the approach of the American Academy of Dermatology. ${ }^{12}$ Others would use the term "spongiotic dermatitis" to describe the same histologic pattern. In fact, this use of "eczema" includes multiple morphologies, ranging from the acute, subacute, and chronic ill-defined lesions present in AD/AE. Moreover, this use of "eczema" is far broader than the initial intent of the term in its original use for acute vesicular or bullous lesions. The term "atopic dermatitis" may be more appropriate, as it frames the multiple disease morphologies with a specific attribution to an atopic phenotype or etiology. On the other hand, many clinicians may use the term "eczema" as a synonym for AD/AE as per the lay public and/or the consensus of the World Allergy Organization. ${ }^{13}$ The World Allergy Organizations proposed that the term eczema should replace the use of terms AD and AE and that AE should refer to eczema in a person of the atopic constitution. ${ }^{13}$ These recommendations have not been widely followed. The disparate use of terminology may be confusing for both clinicians and patients alike.

\subsection{4 | International classification of disease codes}

Importantly, the terms "atopic dermatitis" and "eczema" are associated with different International Classification of Disease (ICD) codes (ICD-9: 691.8 vs 692.9; ICD-10: L20.x vs L30.9, respectively). However, the ICD-9 code 692.9 is meant to be used for unspecified contact dermatitis; the ICD-10 code L30.9 is meant to be used even more broadly for unspecified dermatitis. Thus, when providers type the term "eczema" into an electronic health record, the software may apply incorrect ICD codes. The incorrect use of ICD billing codes might result in lack of reimbursement for certain medications and/or services by payers.

The incorrect use of ICD codes also has major ramifications for epidemiology and health services research of $A D / A E$. A recent study examined the validity of the ICD-9 codes 691.8 (AD) and 692.9 (eczema). ${ }^{14}$ Both codes were commonly used in AD/AE patients, with similar proportions of patients meeting Hanifin and Rajka criteria $^{10}$ and United Kingdom Working Party Criteria ${ }^{15}$ for AD/AE. Ironically, multiple occurrences of the eczema codes were associated with higher specificity (44.9\% vs $23.8 \%$, respectively) and positive predictive value (33.7\% vs $29.9 \%$ ) than multiple occurrences of the $\mathrm{AD} / \mathrm{AE}$ codes. However, the eczema code also included higher rates of contact dermatitis, hand, asteatotic and dyshidrotic eczema, and unspecified rash. This imprecision in coding of $A D / A E$ introduces bias and misclassification error. It is imperative that clinicians be 
more precise in their AD/AE coding. Proper education about correct use of terminology and ICD coding is warranted during medical education and residency training in dermatology and other specialties. This situation might be improved with the new ICD- 11 classification system now being elaborated. ${ }^{16}$

\subsection{Rationale for consensus}

Given the disparate use of different terms for $A D / A E$ and the adverse consequences of this practice, there is an urgent and rational need for harmonized nomenclature for this condition. A single, universally applied name for AD/AE would enable better comparison of the pathophysiology, epidemiology, comorbidities, and socioeconomic burden worldwide. It would also avoid further confusion related to a dichotomous view of $A D / A E$ and eczema in some countries, such as China.

As drug development is increasingly planned and implemented on a global level, a harmonized denomination would avoid a confusion in the assessment of medicines, medical devices, and diagnostic tests. The recruitment of patients for clinical trials requires a clear and consensual nomenclature in order to assure a consistent phenotype across sites and regions. Consistent nomenclature of $A D / A E$ is necessary prior to possible stratifications based on clinical phenotypes and/or biomarkers. The wording is of paramount importance when defining the indications of emerging therapies and formulating a summary of product characteristics.

Moreover, harmonization of nomenclature would improve the study of AD/AE epidemiology and health services research, as well as the interpretation of research by physicians, consumers, and patients, alike. The advantages of the harmonization are obvious in the context of communicating knowledge between the global stakeholders of AD/AE. Last, and possibly most importantly, patients today independently collect information from many resources and need an exact diagnosis to avoid confusion as well as inappropriate guidance and therapy.

\section{2 | METHODS}

The IEC (http://www.eczemacouncil.org/), founded in 2014, is a global nonprofit organization whose membership consists of $77 \mathrm{AD}$ experts from 21 countries on six continents. All councilors and associates are vetted for expertise in the field of $A D$, including research track record. IEC councilors gathered at the American Academy of Dermatology annual meeting in Washington, D.C. in 2016 to review the disparate nomenclature of $A D$ and related challenges. Each topic was discussed by the group, and a team of councilors subsequently reviewed the recent literature to write about the use of terminology for $A D$ in the: i) scientific literature, ii) lay public, and iii) clinical setting. In March 2017, an electronic questionnaire was sent to the IEC membership regarding their preferred nomenclature for AD. Study data were collected and managed using Microsoft Excel.
The survey consisted of three questions: (1) Doyou prefer the use of the atopic prefix (i.e., atopic eczema or atopic dermatitis) or the broader terms eczema or dermatitis? (2) If you answered yes to question 1 , do you prefer the term atopic dermatitis or atopic eczema? Our a priori definition of consensus for questions 1 and 2 was at least $90 \%$ agreement for each question with a response rate of at least $90 \%$. An additional exploratory question was asked, but was not considered for consensus: (3) Regardless of the outcome of this survey, which of the following terms would be acceptable to you (atopic dermatitis/atopic eczema/eczema)?

\section{3 | RESULTS}

Seventy-one of 77 (92.2\%) IEC councilors and associates responded to the survey, with all respondents completing the entire survey. Respondents were from institutions in Australia, ${ }^{1}$ Austria, ${ }^{1}$ Brazil, ${ }^{1}$ Canada, ${ }^{4}$ China, ${ }^{1}$ Denmark, ${ }^{3}$ France, ${ }^{6}$ Germany, ${ }^{12}$ India, ${ }^{1}$ Ireland, ${ }^{2}$ Israel, ${ }^{2}$ Italy, ${ }^{2}$ Japan, ${ }^{4}$ Korea, ${ }^{2}$ the Netherlands, ${ }^{3}$ Spain, ${ }^{1}$ Taiwan, ${ }^{1}$ Tanzania, ${ }^{2}$ UK, ${ }^{5}$ and USA. ${ }^{17}$ Consensus was reached on for question 1 , with 69 of 71 respondents (97.2\%) preferring the atopic prefix (Table 2). Consensus was not reached for question 2 , with 40 respondents (58.0\%) preferring the term atopic dermatitis, $30(43,5 \%)$ preferring atopic eczema, and 1 (1.4\%) invalid response. Sixty-three respondents (88.7\%) and 55 (77.5\%) felt that the terms AD and AE were acceptable, whereas only 11 (15.5\%) felt that eczema was acceptable.

\section{4 | DISCUSSION}

Among a large international group of clinicians and researchers with expertise in $A D$, we reached consensus on the requirement of an

TABL E 2 Survey responses

\begin{tabular}{|c|c|c|c|}
\hline \multirow[b]{2}{*}{ Question } & \multicolumn{3}{|l|}{ Responses } \\
\hline & Yes & No & \\
\hline \multirow{2}{*}{$\begin{array}{l}\text { (1) Do you prefer the use of } \\
\text { the atopic prefix (i.e., atopic } \\
\text { eczema or atopic dermatitis) } \\
\text { or the broader terms eczema } \\
\text { or dermatitis? }\end{array}$} & 69 (97.2\%) & $2(2.8 \%)$ & \\
\hline & $\begin{array}{l}\text { Atopic } \\
\text { dermatitis }\end{array}$ & $\begin{array}{l}\text { Atopic } \\
\text { eczema }\end{array}$ & \\
\hline \multirow{2}{*}{$\begin{array}{l}\text { (2) If you answered yes to } \\
\text { question } 1 \text {, do you prefer } \\
\text { the term atopic dermatitis } \\
\text { or atopic eczema? }\end{array}$} & 40 (58.0\%) & $30(43.5 \%)$ & \\
\hline & $\begin{array}{l}\text { Atopic } \\
\text { dermatitis }\end{array}$ & $\begin{array}{l}\text { Atopic } \\
\text { eczema }\end{array}$ & Eczema \\
\hline $\begin{array}{l}\text { (3) Regardless of the outcome } \\
\text { of this survey, which of the } \\
\text { following terms would be } \\
\text { acceptable to you (atopic } \\
\text { dermatitis/atopic eczema/ } \\
\text { eczema)? }\end{array}$ & $63(88.7 \%)$ & 55 (77.5\%) & 11 (15.5\%) \\
\hline
\end{tabular}


atopic disease prefix, that is, AD or AE. Thus, the consensus of the $\mathrm{IEC}$ is to recommend against the use of the term "eczema" owing to its imprecision, and disparate and confusing real-world use. We were unable to reach consensus for preferred use of the term "atopic dermatitis" vs "atopic eczema," although AD was more commonly preferred and had the highest rate of acceptability. Our recommendation is to standardize the disease nomenclature with use of the terms "atopic dermatitis" or "atopic eczema" in clinical trials, scientific literature, presentations, and patient education.

The consensus to not use the term "eczema" has important ramifications for patient education. "Eczema" is the most common lay term for AD/AE. Currently, clinical history taking and survey-based research studies for AD/AE require use of the term "eczema." Indeed, a recent multicenter validation study found that self- or caregiverreported "eczema or skin allergy" had good sensitivity and positive predictive value. ${ }^{17}$ On the other hand, there are several etiologies of eczematous reactions, and patients may mistakenly assume that they have AD/AE when, in fact, they may have another form of eczema, such as stasis or nummular eczema or contact eczema. Thus, patient counseling for AD/AE must strike a balance between use of familiar lay terminology "eczema" and educating patients about their specific etiology of disease. We therefore recommend that the term "atopic" should be introduced to patients with $A D / A E$, rather than just sharing the diagnostic terms "eczema" or "dermatitis."

The transformation to consistency will not be an easy one. The term "eczema" is shorter and easier to use in conversation with patients (although more difficult to spell), and a shift to AD or AE would require patient discussions to clarify the imprecision of the term "eczema." It may also be difficult for an organization focused on AD that includes "eczema" in its title to change its name to support this unification, especially until a widespread movement is underway to shift to public utilization of the terms "atopic dermatitis" or "atopic eczema." Nevertheless, there is a solid rationale and partial consensus for a more precise name for this disorder by at least using the atopic prefix prior to the terms eczema or dermatitis. We encourage physicians in all specialties and in every country to shift their own use of terminology away from eczema alone to $A D$ or $A E$ in writing, presentations, and discussions with patients and other healthcare personnel as a first step.

\section{CONFLICTS OF INTEREST}

The authors declare no conflict of interest.

\section{RE FE RE NC ES}

1. Andersen RM, Thyssen JP, Maibach HI. Qualitative vs. quantitative atopic dermatitis criteria - in historical and present perspectives. J Eur Acad Dermatol Venereol. 201 6;30:604-618.
2. Rudikoff D, Cohen SR, Scheinfeld N. Atopic dermatitis and eczematous disorders. Boca Raton: CRC Press, Taylor \& Francis Group; 2014.

3. Mugglestone L. The Oxford History of English. Updated Edition. edn. Oxford: Oxford University Press; 2012.

4. Wallach D, Taïeb A, Tilles G, Sociétéfrançaise d'histoire de la dermatologie. Histoire de la dermatite atopique. Issy-les-Moulineaux: Masson;2004.

5. Nast A, Griffiths CE, Hay R, Sterry W, Bolognia JL. The 2016 International League of Dermatological Societies' revised glossary for the description of cutaneous lesions. Br J Dermatol. 2016;1 74:13511358.

6. Kantor R, Thyssen JP, Paller AS, Silverberg Jl. Atopic dermatitis, atopic eczema, or eczema? A systematic review, meta-analysis, and recommendation for uniform use of 'atopic dermatitis'. Allergy. 2016;71:1480-1485.

7. Schultz Larsen F, Hanifin JM. Secular change in the occurrence of atopic dermatitis. Acta Derm Venereol Suppl (Stockh). 1992;176:7-12.

8. Taylor B, Wadsworth J, Wadsworth M, Peckham C. Changes in the reported prevalence of childhood eczema since the 1939-45 war. Lancet. 1984:2:1255-1257.

9. Williams H, Stewart A, vonMutius E, Cookson W, Anderson HR. Is eczema really on the increase worldwide? J Allergy Clin Immunol. 2008;121:947-954.

10. Hanifin J, Rajka G. Diagnostic features of atopic eczema. Acta Derm Venereol. 1980;92:44-47.

11. Xu S, Thyssen J, Paller A, SilverbergJ. Eczema, atopic dermatitis or atopic eczema? An analysis of global search engine trends. Dermatitis. 2017 doi:10.1097/DER.0000000000000273

12. Suarez-Varela MM, Alvarez LG, Kogan MD, et al. Diet and prevalence of atopic eczema in 6 to 7-year-old schoolchildren in Spain: ISAAC phase III. J Investig Allergol Clin Immunol. 201 0;20:469-475.

13. Johansson SG, Bieber T, Dahl R, et al. Revised nomenclature for allergy for global use: report of the Nomenclature Review Committee of the World Allergy Organization, October 2003. J Allergy Clin Immunol. 2004;113:832-836.

14. Hsu DY, Dalal P, Sable KA, et al. Validation of International Classification of Disease Ninth Revision codes for atopic dermatitis. Allergy 2017;72:1091-1095.

15. Williams HC, Burney PG, Hay RJ, et al. The U.K. Working Party's Diagnostic Criteria for Atopic Dermatitis. I. Derivation of a minimum set of discriminators for atopic dermatitis. $\mathrm{Br} J$ Dermatol. 1994;131:383-396.

16. Tanno LK, Calderon MA, Demoly P. New Allergic and Hypersensitivity Conditions Section in the International Classification of Diseases11. Allergy Asthma Immunol Res. 2016;8:383-388.

17. Silverberg I, Patel $\mathrm{N}$, Immaneni S, et al. Assessment of atopic dermatitis using self-report and caregiver report: a multicentre validation study. Br J Dermatol. 2015;173:1400-1404.

How to cite this article: SilverbergJ, ThyssenJP, Paller AS, et al. What's in a name? Atopic dermatitis or atopic eczema, but not eczema alone. Allergy. 2017;00:1-5. https://doi.org/ 10.1111 /all. 13225 